\title{
Delving Deep into the Structural Aspects of the BPro28-BLys29 Exchange in Insulin Lispro: A Structural Biophysical Lesson
}

\author{
Wei $\mathrm{Li}^{*}$
}

July 13, 2020

\begin{abstract}
Insulin lispro was the first fast acting insulin analogue to obtain regulatory approval for therapeutic use. This article puts forward a novel biophysical mechanism where the net impact of the simple B28Pro-B29Lys exchange from regular insulin to insulin lispro is the establishment of a novel set of interfacial electrostatic interactions between Lys28 of insulin lispro and Asp12 of insulin receptor (IR). In addition, a set of structural analysis was presented in this article to further strengthen the binding of insulin lispro to IR, where two polar amino acid residues (Gln51 and Asn74 of insulin lispro) were put forward as two potential targets for site-directed mutagenesis of insulin lispro at its binding interface with IR.
\end{abstract}

${ }^{*}$ Institute of Special Environmental Medicine, Nantong University, No. 9, Seyuan Road, Nantong City, Jiangsu Province, People's Republic of China 


\section{Introduction}

It is almost a century since the discovery of insulin in 1921, a medical triumph which led to it being one of the most intensely studied molecules $[1,2,3,4,5,6]$. While new methods were developed and used to change the structure of regular human insulin to make it more suitable for subcutaneous administration, various insulin analogues were developed with pharmacodynamic and pharmacokinetic features different from that of regular human insulin $[7,8,9,10,11]$, among which insulin lispro was the first fast acting insulin analogue to obtain regulatory approval for therapeutic use in $1996[12,13,14,5,15]$.

Genetically, insulin lispro is engineered in such a way that it has a similar amino acid sequence as the regular insulin but has an exchange of proline (Pro)-lysine (Lys) sequence at positions 28 and 29 in its B chain, leading to the creation of a fast acting B28Lys-B29Pro insulin mutant. Pharmacologically, due to its significantly reduced self-association, insulin lispro offers faster subcutaneous absorption, and begins to exert its effects earlier than its wild-type counterpart [16, 17, 18, 19, 20, 21, 22, 23].

\section{Motivation}

To date, insulin treatment still imposes a challenging regimen and provides suboptimal outcomes for the majority of patients $[24,25,15]$. Moreover, despite continued advancements in the R\&D of fast acting insulin analogues and continued depositions of biomolecular experimental structures in PDB [26], the biophysics underlying still remains not clear from a biomolecular structural point of view. Thus, this article aims to answer: what is the biophysical basis of the BPro28-BLys29 exchange from regular insulin to insulin lispro? what lesson can be learned from it? 


\section{Materials and Methods}

Of this article, all materials and methods are included the Supplementary Materials and Methods section in supplementary file supps.pdf.

\section{Results}

\subsection{Biophysical basis of the simple BPro28-BLys29 exchange from regular insulin to insulin lispro}

Here, a set of structural analysis (Tables 5-9, Figure 2, supplementary file supps.pdf) led to a direct structural observation (Figures 1 and 2) that the net impact of the simple B28Pro-B29Lys exchange from regular insulin to insulin lispro is the establishment of a novel set of interfacial electrostatic interactions between Lys28 of insulin lispro and Asp12 of insulin receptor (IR).

Specifically,

1. there is no interfacial salt bridge or hydrogen bond for Asp12 (of IR) or Pro28 (of regular insulin) or Lys29 (of regular insulin).

2. there is no interfacial salt bridge or hydrogen bond between Asp12 (of IR) and Pro29 (of insulin lispro).

3. there is one interfacial salt bridge (induced by the simple B28Pro-B29Lys exchange) between Asp12 (of IR) and Lys28 (of insulin lispro) (Table 1).

4. there is one interfacial side chain hydrogen bond (induced by the simple B28ProB29Lys exchange) between Asp12 (of IR) and Lys28 (of insulin lispro) (Table 2). 
Of further biophysical interest, the side chain nitrogen atom (carrying one unit of net positive electric charge) of C_LYS_28 is only $4.7 \AA$ away from another side chain oxygen atom (carrying one unit of net negative electric charge) of A_ASP_12 (Table 1 ), which is rather close to the cutoff distance $(4 \AA)$ for salt bridge screening as used previously in [27].

\begin{tabular}{|l|c|c|c|c|c|}
\hline PDB file & Residue A & Atom A & Residue B & Atom B & Distance ( $)$ \\
\hline lispro & C_LYS_28 & NZ & A_ASP_12 & OD1 & 4.687 \\
\hline lispro & C_LYS_28 & NZ & A_ASP_12 & OD2 & 2.702 \\
\hline
\end{tabular}

Table 1: Interfacial salt bridges at vertex B (Figures 3 and 4) of the electrostatic triangle in the complex structure of IR and insulin lispro. In this table, the residue naming scheme is Chain ID_residue name_residue number, and '.pdb' is not included in the PDB file.

\begin{tabular}{|l|l|l|l|c|c|c|}
\hline PDB file & Acceptor (A) & Donor (D) & Hydrogen $(\mathrm{H})$ & D-A $(\AA)$ & H-A $(\AA)$ & $\angle A D H\left(^{\circ}\right)$ \\
\hline lispro & OD2, A_ASP_12 & NZ, C_LYS_28 & HZ2, C_LYS_28 & 2.70 & 1.81 & 22.61 \\
\hline
\end{tabular}

Table 2: Interfacial side chain hydrogen bond at vertex B (Figures 3 and 4) of the electrostatic triangle in the complex structure of IR and insulin lispro. In this table, the residue naming scheme is Chain ID_residue name_residue number, $\angle A D H$ represents the angle formed by acceptor $(\mathrm{A})$, donor $(\mathrm{D})$ and hydrogen $(\mathrm{H})(\angle A D H)$, and '.pdb' is not included in the PDB file.

With a close inspection of the structural model of IR in complex with insulin lispro (supplementary file lispro.pdb), an electrostatic interaction triangle was found to sit at the binding interface of IR and insulin lispro, which constitutes an even more favourable contribution towards insulin-IR interfacial structural stabilization, as illustrated in Figures 3 and 4. 


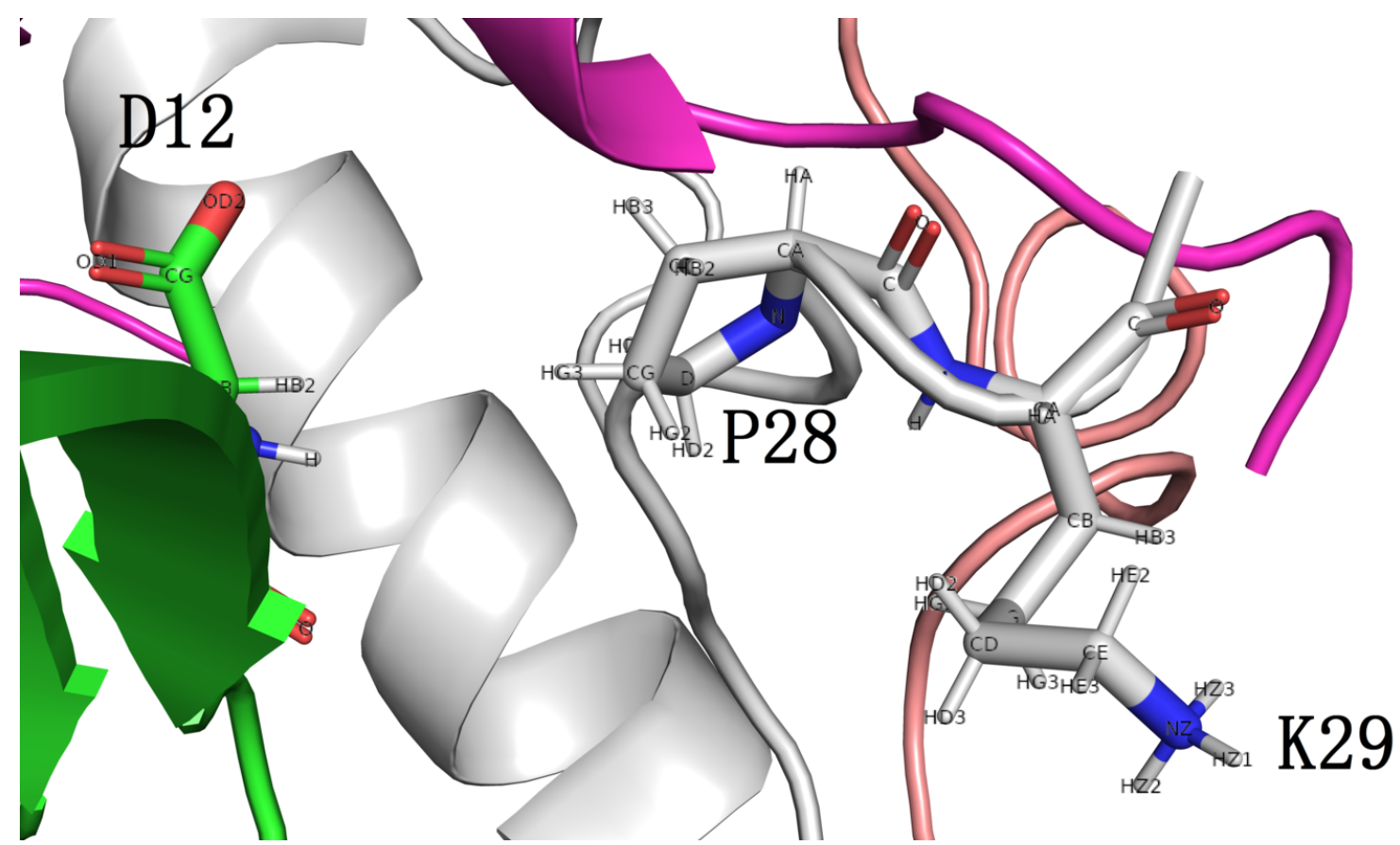

Figure 1: The spatial locations of Asp12 (of IR, green cartoon), Pro28 (of regular insulin, gray cartoon) and Lys29 (of regular insulin, gray cartoon) in the complex structure of regular insulin bound to IR (PDB ID: 6SOD). In this figure, all atoms of the three residues are labelled with their respective names, and the side chain nitrogen atom of Lys29 is as far as 13.9, 10.9, and $14.3 \AA$ away from the two side chain oxygen atoms and the backbone oxygen atom, while the side chain carbon atoms of Pro28 are as far as 6 and $6.8 \AA$ away from the the two side chain oxygens and backbone oxygen atom of Asp12. 


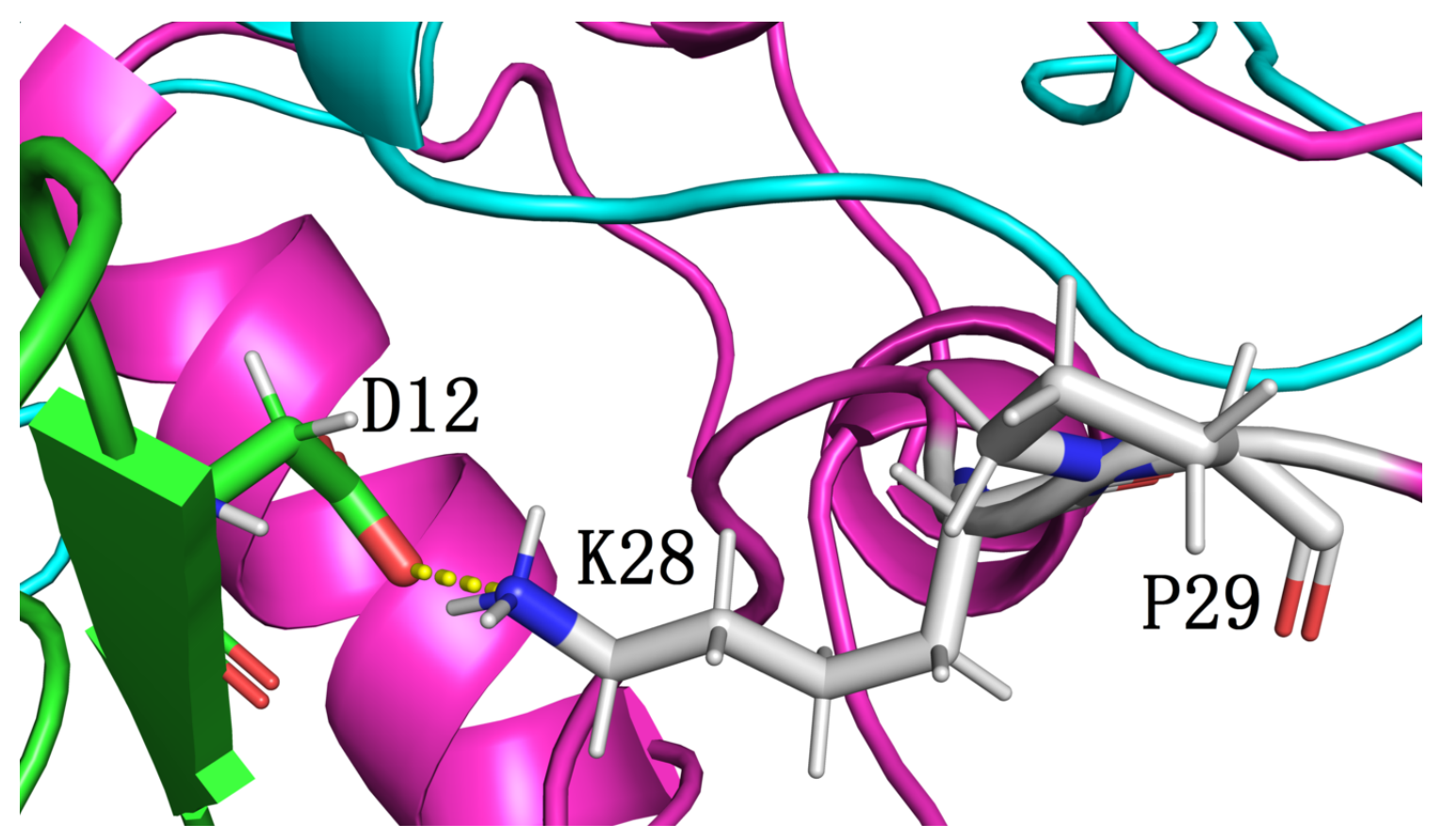

Figure 2: The spatial locations of Asp12 (of IR, green cartoon), Lys28 (of insulin lispro, purple cartoon) and Pro29 (of insulin lispro, purple cartoon) in the complex structure of insulin lispro bound to IR (supplementary file lispro.pdb). In this figure, the side chain nitrogen atom of Lys28 is only 2.7, 3.1 and $4.7 \AA$ away from the two side chain oxygen atoms and the backbone oxygen atom of Asp12, allowing the establishment of a set of strong interfacial electrostatic interactions (salt bridging, dipole-dipole interaction and side chain hydrogen bonding) between insulin lispro and IR. 


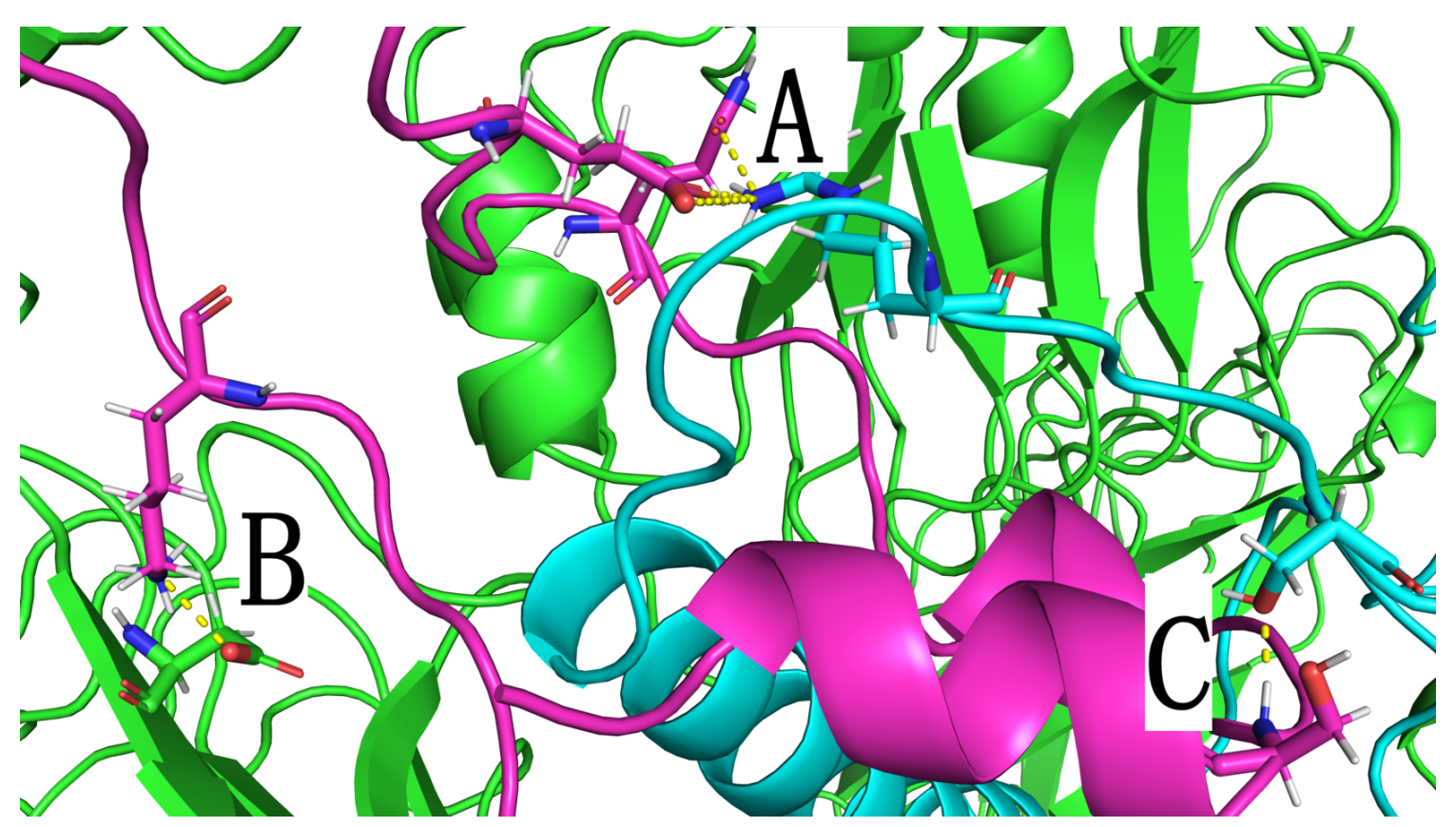

Figure 3: An electrostatic interaction triangle $(\triangle A B C)$ stabilizes the insulin lispro-IR complex structure (supplementary file lispro.pdb). Of the $\triangle A B C$ in this figure, all details of the three insulin lispro-IR-stabilizing vertices are listed in Tables 10-14 in supplementary file supps.pdf, all interfacial electrostatic interactions are indicated with yellow dotted lines beside the three letters A, B and C. In this figure, IR is shown as green cartoon, chains A and B of insulin lispro in cyan and purple cartoon, respectively. 


\section{Q51+R720+E47}

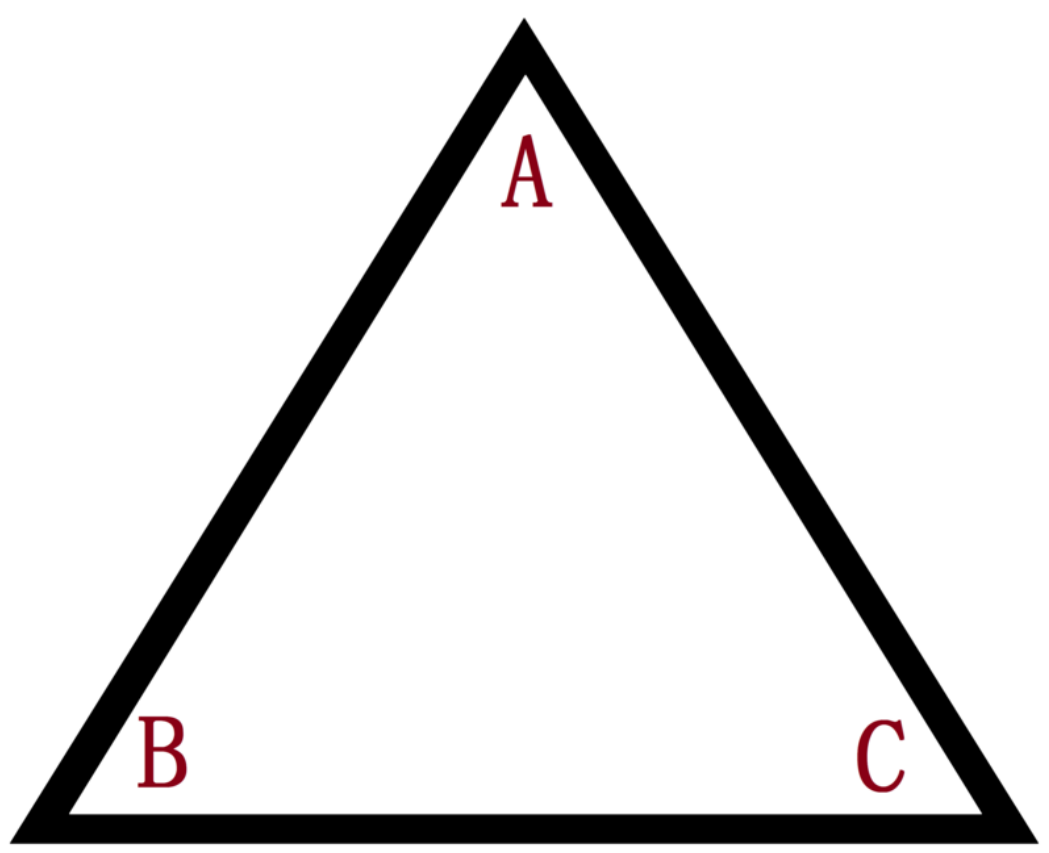

K28+D12

S724+S65

Figure 4: An electrostatic interaction triangle $(\triangle A B C$, Figure 3) stabilizes the complex structure of insulin lispro and IR (supplementary file lispro.pdb). Of the $\triangle A B C$ in this figure, all details of the three insulin lispro-IR-stabilizing vertices ( $\mathbf{A}$, B and C, the same as those in Figure 3) are listed in Tables 10-14 in supplementary file supps.pdf. 


\section{Conclusion}

Incorporating currently available structural data, this article puts forward a biophysical mechanism where the net impact of the simple B28Pro-B29Lys exchange from regular insulin to insulin lispro is the establishment of a novel set of interfacial electrostatic interactions between Lys28 of insulin lispro and Asp12 of IR at their

binding interface (Figures 1 and 2), which constitutes an energetically favourable contribution towards the binding of insulin lispro and IR and towards their interfacial structural stabilization, too.

In addition to the biophysical basis discussed above, this article also puts forward a set of structural analysis towards the design of novel insulin analogues to enhance the binding affinity between insulin and IR, which is to be discussed as below.

\section{How insulin-IR binding can be improved using mutation-induced interfacial electrostatic pertur- bation: a structural biophysical lesson}

Another insulin analogue, peglispro [28, 29, 30, 31] was derived by covalent attachment of a linear $20 \mathrm{kD}$ polyethylene polymer to the LysB28 side chain in insulin lispro. With such modification, extended action of insulin peglispro was achieved through increased hydrodynamic size of the analogue owing to the PEG conjugate [15, 32]. This increase in size results in slower subcutaneous absorption, which contributes to the appreciable prolongation of the half-life of insulin peglispro $[15,32]$.

However, as mentioned above, insulin treatment to date is still a challenging field and provides sub-optimal outcomes for the majority of patients $[24,25,15]$. Here, 
with a set of electrostatic interaction analysis in supplementary file supps.pdf, this article puts forward two structural clues for the design of novel insulin analogues with higher binding affinity to IR than insulin lispro [33, 34]. For examples, two side chain hydrogen bonds already exist at the insulin-IR complex structural interface (Table 3). On top of the two hydrogen bonds (Table 3), site-directed mutagenesis [34] (Gln51Glu or Asn74Asp, Table 3) of insulin lispro can be generated to create stronger charge-charge interactions at the insulin-IR complex structural interface, where two sets of novel interfacial salt bridges can be created between Arg717 and Asp74, and between Arg720 and Glu51, on top of the two side chain hydrogen bonds which already exist at the insulin lispro-IR interface (Table 3).

\begin{tabular}{|l|l|l|l|c|c|c|}
\hline PDB file & Acceptor (A) & Donor (D) & Hydrogen (H) & D-A $(\AA)$ & H-A $(\AA)$ & $\angle A D H\left(^{\circ}\right)$ \\
\hline lispro & OXT, D_ASN_74 & NH1, A_ARG_717 & HH12, A_ARG_717 & 2.67 & 1.77 & 21.20 \\
\hline lispro & OE1, C_GLN_51 & NH2, B_ARG_720 & HH22, B_ARG_720 & 2.71 & 1.86 & 26.51 \\
\hline
\end{tabular}

Table 3: Two interfacial side chain hydrogen bonds for IR in complex with insulin lispro. In this table, the residue naming scheme is Chain ID_residue name_residue number, $\angle A D H$ represents the angle formed by acceptor (A), donor (D) and hydrogen $(\mathrm{H})(\angle A D H)$. With site-directed mutants (Gln51Glu or Asn74Asp).

Biophysically, these site-directed mutagenesis for Asn74 and Gln51 (Gln51Glu or Asn74Asp) are able to strengthen the binding affinity between IR and novel insulin lispro analogues, in a manner similar to the biophysical mechanism discussed above, where the simple B28Pro-B29Lys exchange from regular insulin to insulin lispro strengthens the binding of insulin lispro to IR. 


\section{References}

[1] Nicol D, Smith L. Amino-Acid Sequence of Human Insulin. Nature. 1960 Aug;187(4736):483-485.

[2] Derewenda U, Derewenda Z, Dodson GG, Hubbard RE, Korber F. Molecular Structure of insulin: The insulin monomer and its assembly. British Medical Bulletin. 1989;45(1):4-18.

[3] Adams MJ, Blundell TL, Dodson EJ, Dodson GG, Vijayan M, Baker EN, et al. Structure of Rhombohedral 2 Zinc Insulin Crystals. Nature. 1969 Nov;224(5218):491-495.

[4] Bentley G, Dodson E, Dodson G, Hodgin D, Mercola D. Structure of insulin in 4-zinc insulin. Nature. 1976 May;261(5556):166-168.

[5] Insulin Lispro. New England Journal of Medicine. 2003 Oct;349(15):1487-1487.

[6] Uchikawa E, Choi E, Shang GJ, Yu HT, Bai XC. Cryo-EM structure of full-length insulin receptor bound to 4 insulin. 3D refinement was focused on the top part of the receptor complex. Worldwide Protein Data Bank; 2019.

[7] Sharma AK, Taneja G, Kumar A, Sahu M, Sharma G, Kumar A, et al. Insulin analogs: Glimpse on contemporary facts and future prospective. Life Sciences. 2019 Feb;219:90-99.

[8] Miikkulainen K, Caruso A, Mast O, Zhang R, Borisenko O. Systematic literature review of use of blood glucose monitoring in phase III clinical studies of insulin analogs. BMC Endocrine Disorders. 2016 May;16(1). 
[9] Zhang B, Liu M, Arvan P. Behavior in the Eukaryotic Secretory Pathway of Insulin-containing Fusion Proteins and Single-chain Insulins Bearing Various B-chain Mutations. Journal of Biological Chemistry. 2002 Nov;278(6):3687-3693.

[10] Bode B. Comparison of Pharmacokinetic Properties, Physicochemical Stability, and Pump Compatibility of 3 Rapid-Acting Insulin Analogues-Aspart, Lispro, and Glulisine. Endocrine Practice. 2011 Mar;17(2):271-280.

[11] Home PD. The pharmacokinetics and pharmacodynamics of rapid-acting insulin analogues and their clinical consequences. Diabetes, Obesity and Metabolism. 2012 Mar;14(9):780-788.

[12] Kaiserman K, Jung H, Benabbad I, Karges B, Polak M, Rosilio M. 20 Years of insulin lispro in pediatric type 1 diabetes: a review of available evidence. Pediatric Diabetes. 2016 Jul;18(2):81-94.

[13] Baeshen NA, Baeshen MN, Sheikh A, Bora RS, Ahmed M, Ramadan H, et al. Cell factories for insulin production. Microbial Cell Factories. 2014 Oct;13(1).

[14] Walsh G, Murphy B, editors. Biopharmaceuticals, an Industrial Perspective. Springer Netherlands; 1999.

[15] Zaykov AN, Mayer JP, DiMarchi RD. Pursuit of a perfect insulin. Nature Reviews Drug Discovery. 2016 Mar;15(6):425-439.

[16] Howey DC, Bowsher RR, Brunelle RL, Rowe HM, Santa PF, Downing-Shelton J, et al. [Lys(B28), Pro(B29)]-human insulin: Effect of injection time on postprandial glycemia. Clinical Pharmacology \& Therapeutics. 1995 Oct;58(4):459-469. 
[17] Korn M, Wohlfart P, Gossas T, Kullman-Magnusson M, Niederhaus B, Dedio J, et al. Comparison of metabolic and mitogenic response in vitro of the rapidacting insulin lispro product SAR342434, and US- and EU-approved Humalog(R. Regulatory Toxicology and Pharmacology. 2019 dec;109:104497.

[18] Pala L, Dicembrini I, Mannucci E. Continuous subcutaneous insulin infusion vs modern multiple injection regimens in type 1 diabetes: an updated meta-analysis of randomized clinical trials. Acta Diabetologica. 2019 apr;56(9):973-980.

[19] Hajjaji IM, Eshwihdi N, Barrowman N. Comparison of analog insulin mix 50:50 with human insulin mix 30:70 in persons with type 2 diabetes during Ramadan. International Journal of Clinical Practice. 2019 apr;73(6):e13348.

[20] López-Tinoco C, Jiménez-Blázquez JL, Larrán-Escandón L, del Mar RocaRodríguez M, Bugatto F, Aguilar-Diosdado M. Effect of Different Insulin Therapies on Obstetric-Fetal Outcomes. Scientific Reports. 2019 nov;9(1).

[21] Qasem RJ, Aldawsari AS, Almutairi FE, Alsadoon AS. Identification of recombinant human insulin and biosynthetic insulin analogues by multiplexed targeted unlabeled mass spectrometry of proteotypic tryptic peptides. Journal of Pharmaceutical and Biomedical Analysis. 2019 aug;172:357-363.

[22] Janež A, Guja C, Mitrakou A, Lalic N, Tankova T, Czupryniak L, et al. Insulin Therapy in Adults with Type 1 Diabetes Mellitus: a Narrative Review. Diabetes Therapy. 2020 jan;11(2):387-409.

[23] Bilotta F. Short acting insulin analogues in intensive care unit patients. World Journal of Diabetes. 2014;5(3):230. 
[24] Hirsch IB. Insulin Analogues. New England Journal of Medicine. 2005 Jan;352(2):174-183.

[25] Home P, Riddle M, Cefalu WT, Bailey CJ, Bretzel RG, del Prato S, et al. Insulin Therapy in People With Type 2 Diabetes: Opportunities and Challenges? Diabetes Care. 2014 May;37(6):1499-1508.

[26] Berman H, Henrick K, Nakamura H. Announcing the worldwide Protein Data Bank. Nature Structural \& Molecular Biology. 2003 dec;10(12):980-980.

[27] Li W. How do SMA-linked mutations of SMN1 lead to structural/functional deficiency of the SMA protein? PLOS ONE. 2017 jun;12(6):e0178519.

[28] Sanyal A, Cusi K, Hartman ML, Zhang S, Bastyr EJ, Bue-Valleskey JM, et al. Cytokeratin-18 and enhanced liver fibrosis scores in type 1 and type 2 diabetes and effects of two different insulins. Journal of Investigative Medicine. 2017 Nov;66(3):661-668.

[29] Hirose T, Cai Z, Yeo KP, Imori M, Ohwaki K, Imaoka T. Open-label, randomized study comparing basal insulin peglispro and insulin glargine, in combination with oral antihyperglycemic medications, in insulin-nave Asian patients with type 2 diabetes. Journal of Diabetes Investigation. 2017 May;9(1):100-107.

[30] Muñoz-Garach A, Molina-Vega M, Tinahones FJ. How Can a Good Idea Fail? Basal Insulin Peglispro [LY2605541] for the Treatment of Type 2 Diabetes. Diabetes Therapy. 2016 Nov;8(1):9-22.

[31] Pettus J, Cavaiola TS, Tamborlane WV, Edelman S. The past, present, and future of basal insulins. Diabetes/Metabolism Research and Reviews. 2015 Nov;32(6):478-496. 
[32] Caparrotta TM, Evans M. PEGylated insulin Lispro, (LY2605541)-a new basal insulin analogue. Diabetes, Obesity and Metabolism. 2013 Sep;16(5):388-395.

[33] Ampudia-Blasco FJ. Biosimilars and Novel Insulins. American Journal of Therapeutics. 2020;27(1):e52-e61.

[34] Jubb HC, Pandurangan AP, Turner MA, Ochoa-Montaño B, Blundell TL, Ascher DB. Mutations at protein-protein interfaces: Small changes over big surfaces have large impacts on human health. Progress in Biophysics and Molecular Biology. 2017 Sep;128:3-13. 